\title{
A Model for Analyzing the Efficiency of SEO and Adwords Campaigns in the Romanian and American Online Media
}

\author{
Irina Olimpia Susanu \\ irinasusanu@gmail.com \\ Daniela Damian \\ danah2030@yahoo.com \\ Constantin Avram \\ Robert Rusu \\ robert.rusu@,ugal.ro \\ Dunarea de Jos University of Galati, Romania
}

\begin{abstract}
Focusing and investing in SEO and AdWords campaigns is the secret weapon underlying the acquisition of supremacy on a scene of a tacit war on the market. Thus, if the presence in the online environment was not important in the past, it has now become crucial. The purpose of this paper is to identify the link between the campaign budget, its period, the position of the main competitors and the elements used so that the results are optimal regardless of the type of organization or its geographical area. In this sense, an analysis model has been developed that highlights the link between these elements and the obtained performance. The methodology used focused on monitoring and quantifying the performance of SEO and AdWords campaigns for two US and one Romanian websites, and the quantification of performance was done using Balanced ScoreCard.
\end{abstract}

Keywords: SEO, Adwords, Optimization, Cost Per Click, Balanced Scorecard

\section{Introduction}

Internet is a wide community including millions of users and it is also named as one of today's most important communication tools. This wide community is also known as an important customer potential for everyone who thinks about business works based on Internet environment. Internet has become new channel of the business area in the modern world in which it is not possible to reach such a wide volume by using traditional selling channels (Yalçna N., Köseb, U., 2010) SEO is a the science of customizing elements of your web site to achieve the best possible search engine ranking (Ledford J.L., 2008). Search engine optimization (SEO) generally describes the use of computing systems for running com-puting processes that collect, store and analyze search engine data in order to provide recommendations to improve visibility of a website or a webpage in search engines (Park L.S, YuSammy J., Liao Y., 2012). As a result of the high expected quality on the organic side, consumers begin their search with an organic click. Although SEO can improve consumer welfare and the payoff of high-quality sites, we find that the search engine's revenues are typically lower when advertisers spend more on SEO and thus less on sponsored links. Modeling the impact of the minimum bid set by the search engine reveals an inverse U-shaped relationship between the minimum bid and search engine profits, suggesting an optimal minimum bid that is decreasing in the level of SEO activity. (Berman, R., Katona, Z., (2013).

The purpose of this paper is to identify the link between the campaign budget, the period, the position of the main competitors and the elements used so that the results are optimal regardless of the type of organization or geographical area. 


\section{Methodology}

The methodology used focused on monitoring and quantification of the performance of SEO and AdWords campaigns for two U.S. and onewebwebwebweb sites. The two websites belong to the same niche, both selling clothing and footwear, but in order to perform a comparative analysis, the two websites were selected from totally different geographical areas. Thus, for both websites, SEO and AdWords campaigns were designed and implemented to highlight the process of implementing campaigns and quantifying performance.

To enter the online environment, organizations need to go through a series of phases and make practical use of online promotion. Thus, the analyzed organizations have gone through the following stages:

- Creating a website (US)

- Promoting the website on social networks such as Facebook and Twitter (both)

- Promoting the website using Search Engine Optimization (SEO) campaigns to get the top positions in search engines such as Google, Bing and Yahoo (both)

- Promote your website using paid ads (AdWords campaigns).

\section{Specific campaigns, SEO and Adwords}

\subsection{SEO campaign}

Implementing an SEO campaign is one of the actions taken by both organizations to promote their website in the online environment, and both organizations have called on a specialized company to design and implement the SEO campaign. The strategic variations were different because the American website was at the beginning and the SEO strategy approached was aggressive and lasting, while for the Romanian web site, which already enjoys good results in the search engines, the strategy was a consolidation and slightly improving the position in the search engines, so the SEO process was a temperate one.

The purpose of the SEO campaign for the American website was to increase visibility in search engines with the following objectives:

- Get the first position in the search engines for certain keywords

- Get monthly sales of US \$2,500

- Get 10 monthly orders

While for the Romanian website the aim was to improve the position in the search engines; with objectives such as:

- Increase sales by $20 \%$

- Get a monthly business figure of 10,000 lei

Increase in customer numbers by $20 \%$

The SEO campaign designed for the American website has been an aggressive one to promote higher search engine rankings for relevant keywords. Campaign implementation was done for the local search engine version, having the .COM affiliated with the United States of America, respectively .ro for Romania. Thus, campaigns have been deciphered predominantly for the google.com search engine, ie .ro based on the information that is used by about $90 \%$ of the internet users.

Both SEO campaigns included the following services:

- On-page optimization (of the website itself)

- Off-page optimization (link creation)

- Writing original items and short descriptions

- Identify the most relevant keywords

Campaign Duration and Allotted Budget Aggressive SEO campaign is specific to the US website, it has been implemented for a period of 5 months, and the one for the Romanian website, for 3 months, during which it is expected to be the first position in the Google search engine for all the selected keywords. Thus, the SEO campaign for the US website was divided into 5 sub-campaigns of SEO, each sub-campaign running over a calendar month, with a total budget of $\$ 1,000$, divided equally for each of the 5 SEO subcampaigns. Due to the fact that the 
Romanian web site already had favorable results in the online environment, the SEO campaign took place over 3 calendar months, and the budget was narrower, to each SEO subcamp for a calendar month, amounting to $\$ 200$.

The results obtained

The positions obtained in the search engines

The SEO campaign designed and implemented for the American website has been successful as it has led to the goal and two of the objectives. Thus, following the implementation of the SEO campaign, the visibility of the American website in search engines increased, as well as getting 10 monthly orders amounting to more than US \$2,500 a month.

As far as the goal that was not achieved as a result of the SEO campaign, namely getting the first position in the search engines for all the promoted keywords, notable improvements were achieved, but they did not coincide with the original target in sight. At the same time, the SEO campaign favored the overriding online competition of the main competitors, the desirable goal of any organization.

In the case of the Romanian website, the SEO campaign has been effective and has helped to improve search engine rankings for selected keywords

Traffic registered Simultaneously with improving search engine positions, there has also been a major increase in traffic to the website. This is because the ability of a website to be accessed by users increases when it has superior positions in search engines. In our case, the improvement in the volume of traffic received was considerable, especially considering that the website was barely created at the time of implementing the SEO campaign.

In conclusion, the SEO campaign has had positive effects on the US website, leading to both improving search engine positions and increasing traffic volumes.

In most cases, changes in search engine positions directly affect the traffic that a website receives. Thus, the more a website has better positions in the search engine, the higher the traffic volume. Of course, it is desirable that the traffic that a website receives is targeted, so those who access the web site are interested and interested in the products that it sells. In conclusion, the SEO campaign created and implemented for the Romanian website has led to the achievement of the goal and one of the established objectives and can be considered partially effective. Thus, the implementation of the SEO campaign has succeeded in improving the positions of the Romanian website in the search engines, as well as increasing the sales by $20 \%$. The other objectives envisaged have proved to be very ambitious, so they have not been achieved.

\subsection{AdWords Campaign}

As a result of the success of the SEO campaign, the US website has turned its attention to another online advertising tool, namely Google AdWords. As with the SEO campaign, AdWords has been created and implemented by a specialized firm. The AdWords campaign has been done over the course of 45 days and the budget was $\$ 2000$.

The goal of the AdWords campaign was to increase the visibility of the American website in the online environment, with the following goals:

- Get the maximum number of clicks possible

- Get the maximum number of possible conversions

- Get a CPC in the range $\$ 0.5-\$ 1$

As with the US website, the Romanian website also opted for creating and implementing an AdWords campaign to promote the website in the online environment. The campaign has gone through over 40 days, with the budget being $\$ 1500$.

The goal of the AdWords campaign was to enhance visibility in the online environment with the following goals:

- Get the maximum number of clicks possible;

- Get the maximum number of possible conversions;

- Get a $\$ 0.5$ Valuable CPC. 


\section{AdWords Campaign Mathematical Modeling}

For an AdWords campaign to be effective, it is primarily necessary that the ad's website be visible and displayed in Google's search engine results. As far as the websites under review are concerned, the implementation of the AdWords campaign has encountered the following obstacle: their ads compete with the ads of two other websites to get a 2 of the available positions that Google displays in this case. Thus, it was necessary to establish a mathematical model by which the sites analyzed analyze one of the 2 available positions. At the heart of this process was the mechanism according to which the leading bidder is the leading bidder, and the second position of the firm with the value of the next bid. I wanted to identify some variables that influence the value of the bidding for each thread, as well as a calculation formula to determine this value. Thus, the main variables considered for calculating the bid were: • The maximum perclick cost that each company was willing to pay; - The Quality Score or Quality of paid advertising of each business.

Depending on these variables, the display order or the hierarchy of the payouts paid by the watched lines was calculated as follows:

$\mathrm{N}=$ number of websites $\mathrm{N}=3$

$\mathrm{K}=$ Positions available for displaying paid ads $\mathrm{K}=2$

$\mathrm{Bi}=$ Maximum Cost Per Click

Qi $=$ Quality Score

$\mathrm{Ri}=$ bid value

$\mathrm{F} 1=$ Competitor website no. 1

F2 = Competitor website no. 2

$\mathrm{F} 3=$ the website that is under analysis

Making this information in a mathematical model is the following: $\mathrm{K}<\mathrm{N}$

$$
\mathrm{Ri}=\mathrm{Bi} * \mathrm{Qi}, \mathrm{i}=1.3
$$

By analyzing the individual bid values of each website, we can see that the 2 advertisements that will appear in the results of the paid advertisements are that of the website analyzed and the contestant's website no. 2 due to the fact that they recorded the highest bid values. As far as the website under review is concerned, it has placed itself on the first position in the paid ads, thus increasing the chances of success of the campaign according to the following relationship:

$\alpha 1>\alpha 2>\alpha 3$

$\mathrm{ai}=$ the probability of an ad being accessed

In the case of the Romanian web site, it can be seen that this date has not yet achieved the first position in the paid advertising system but the second available position. Thus, the bidding hierarchy of the three websites was as follows:

1. F2 - Competitor website no. 2

2. F3 - the website under analysis

3. F1 - Competitor website no. 1

After calculating each bid, we can conclude that the success of positioning an ad in the AdWords system is due to the optimal combination of the quality of the ad and the maximum cost an organization is willing to pay for its ad. This optimal combination is the first step in implementing an effective AdWords campaign.

\section{Quantification of performance using Balanced ScoreCard s}

In order to determine the effectiveness or otherwise the inefficiency of implementing AdWords campaigns for the two websites, the quantification of the indices in question was done using the 
Balanced Sco-reCard application. Performance indices in view were grouped into two different categories according to their nature, namely: economic indices and technical indices.

\subsection{Economic indices}

- budget allocated to the campaign

- The fee charged by the business that implemented and edited the AdWords campaign

- Profit or loss recorded

Budget allocated to the campaign

The US company had a budget for the AdWords campaign ranging from \$ 2000 to \$ 2500 , with the goal of investing only the lower limit.

The company that dealt with campaign implementation expected it to be worth $\$ 500$, but the favorable campaign outcome has led the specialized firm to earn just $\$ 300$.

The Romanian company has allocated to the AdWords campaign a budget of $\$ 1,500$, which was consumed during the 40 days of the campaign, and the implementation firm's commission was $\$ 200$.

Profit or loss

The US firm has set an ambitious goal to turn every possible click into a conversion (selling), predicting a profit of $\$ 428,550$ at a price of $\$ 150$ traded products.

The Romanian company has set itself the goal of making a profit as high as possible by transforming each click received into a sale. Although this goal has proven to be extremely ambitious, it still relies on a $\$ 100,000$ profit at a $\$ 60$ average.

\subsection{Technical Indices}

- the number of clicks you have received

- the number of conversions recorded

- Average Per Click Cost

Number of clicks

The US firm has set the maximum number of possible clicks (2857 clicks) based on the allocated budget $(\$ 2000)$ and the average per-click cost of $\$ 0.7$.

In the case of the Romanian site, they have proposed to get the maximum number of clicks, ie 1667 clicks, provided that the entire $\$ 1500$ budget would have been consumed for the $\$$ 0.9 average CPC.

Number of conversions

Both companies have proposed to transform each click received into a conversion, but most of the times, such a goal proves to be extremely ambitious.

Thus, taking into account the maximum number of clicks that the Romanian site could achieve by 1667 clicks, the goal was to get the same number of conversions (sales).

Average Per Click Cost

The Per Click average cost of the US campaign was calculated by arithmetic average of all campaign keywords in the $\$ 0.2$ and $\$ 1.5$ range, giving an average CPC of $\$ 0.7$. The average $\mathrm{CPC}$ of the Romanian site was $\$ 0.5$, but the average CPC could not be lowered below $\$ 0.9$. The values of the previous indices have been entered in the BSC application, the results obtained for the US web site are shown in Figure no. 1.

Analyzing the information provided by Balanced ScoreCard Designer, it can be seen that this AdWords campaign recorded a performance of $44.03 \%$. Although this value is not close to the upper limit, the campaign was effective, bearing in mind that this has led to two of the goals in mind: obtaining a CPC of between $\$ 0.5-\$ 1$ and getting the most clicks. The campaign also helped to increase the visibility of the website, promote it online, and make profit. Analyzing the values provided by the BSC application, it can be noticed that for the Romanian website the AdWords campaign recorded a performance of $52.49 \%$, which means overall, the campaign was efficient and performing. It can also be noticed that the highest performance was registered by 
eco-nomic indicators $(56.9 \%)$, while the technical indicators achieved a performance of only 48.08\%.In conclusion, the AdWords campaign has been effective on the Romanian website, as it has led to the achievement of the original goal as well as to achieving one of the objectives.

\section{Conclusions}

The overwhelming conclusion of this paper is that by implementing SEO and AdWords campaigns to promote an online business, it can be projected to the heart of the performance. Thus, SEO and AdWords campaigns are used on a wider scale thanks to indisputable benefits such as: improving website positions in search engines, increasing traffic, and boosting sales with profits.

These online marketing tools provide organizations with the opportunity to increase their visibility in the online environment and to promote their websites and products and / or services, thus gaining the ultimate competitive advantage that will help achieve the highest level of efficiency and performance. Under the assertion in the online environment, a comparative analysis can be made between the US and the Romanian website in order to highlight the similarities and differences between the approaches taken for each of the two websites.

Thus, for both the US and the Romanian web site, SEO and AdWords campaigns have been created, implemented and maintained with the stated purpose of increasing the visibility of these two websites in the online environment. As for the SEO campaign, the efforts of the US site were more intense and sustained, the allocated budget was higher $(\$ 1,000)$, and the duration of the campaign was also longer ( 5 months). As for the SEO campaign for the US website, it was more moderate, the allocated budget was lower $(\$ 600)$, and the duration was also more limited (3 months). Despite the differences, SEO campaigns have been effective in both cases and have led to the improvement of the visibility of both websites in the online environment.

As for the AdWords campaign, the US company invested \$2,000 in stakes over 5 months, paid a $\$ 300$ commission and earned $\$ 39,400$. In the case of the Romanian website, $\$ 1,500$ was invested in the AdWords campaign, they paid a \$ 200 commission and earned a \$13,800 profit. Research limitations / implications

Only the two web sites for a specific query were analyzed. Analyzing more web sites and comparing them with similar studies in different competition would provide more concrete results.

Acknowledgement

This work was supported by a grant of the Romanian Ministry of Research and Innovation, CCCDI - UEFISCDI, project number PN-III-P1-1.2-PCCDI-2017-0800 / 86PCCDI/2018 FutureWeb, within PNCDI III.

\section{References}

1. Ledford, J.L., (2008) "Search engine optimization bible", Second Edition, Wiley Publishing, Indianapolis, $2008, p .34$

2. Michael P. Evans, (2007) "Analysing Google rankings through search engine optimization data", Internet Research, Vol. 17 Issue: 1, pp.21-37, bttps:// doi.org/10.1108/10662240710730470

3. Park L.S, Y uSammy J., Liao Y. (2012) "Search engine optimization for category specific search results", bttps:/ /patents.google.com/patent/US9116994B2/en

4. Yalcna N., Köseb, U. (2010) "What is search engine optimization: SEO?", Procedia Social and Behavioral Sciences 9, p. 487-49

5. Berman, R., Katona, Z., (2013), "The Role of Search Engine Optimization in Search Marketing", Marketing Science, Volume 32, Issue 4, p. 533-678 